March 18-20, 2008, Faculty of Engineering, Tanta Univ., Egypt

\title{
Motion Artifact Suppression in MRI Using k-Space Overlap Processing
}

\author{
Yasser M. Kadah \\ Biomedical Engineering Department, Cairo University, Egypt (E-mail: ymk@k-space.org)
}

\begin{abstract}
Starting from the general assumption of rigid body motion, we consider the case when the acquisition of the $\mathrm{k}$-space is in the form of bands of finite number of lines arranged in a rectilinear fashion to cover the k-space area of interest. We consider cases with an averaging factor of at least 2. Instead of acquiring a full $\mathrm{k}$-space of each image and then average the result, we develop a new acquisition strategy based on acquiring the k-space in consecutive overlapped bands. In case of no motion, this overlap can be used as the second acquisition. On the other hand, when motion is encountered, such overlap can be used to substantially reduce motion artifacts in the resultant image. This is achieved by utilizing the overlap area to estimate the motion, which is then taken into consideration in image reconstruction. We demonstrate the success of this approach using both numerical simulations as well as real data acquired from a human volunteer. The new method has the potential for practical applications to make more efficient use of MRI scanners and making the scan time lower allowing for more comfort to the patient.
\end{abstract}

Keywords: Motion artifact, magnetic resonance imaging, artifact suppression, navigator echo

\section{INTRODUCTION}

Accurate diagnosis in medical procedures has become widely attainable by the advent of the different medical imaging modalities. Among those, magnetic resonance imaging (MRI) is currently one of the most promising non-invasive diagnostic tools in medicine. In addition to its ability to produce anatomical images of remarkable detail and contrast, it can be used to visualize vascular structures, measure blood flow and perfusion, detect neural activation, and identify the metabolic information of different areas in the acquired images. Also, its inherently volumetric acquisition permits slices at different angles to be computed easily which can be advantageous in many applications.

One of the major problems in the present MRI technology is its susceptibility to substantial artifacts when motion occurs during the image acquisition time. Even though fast acquisition methods such as echoplanar imaging (EPI) and spiral imaging provide a solution to this problem for some applications, these techniques are extremely sensitive to magnetic field inhomogeneity effects as compared to regular scanning methods and have a generally low signal-to-noise ratio. This makes it difficult to accurately correlate the generated images with the physical anatomy because of geometric distortion in addition to more profound signal loss within the areas of large susceptibility mismatches. Moreover, when these imaging sequences are used in such applications as functional magnetic resonance imaging (fMRI), where a set of slices are acquired repeatedly, patient motion persists in the form of low detection of activation sites as a result of misregistration of images along the sequences [1].

Due to practical constraints from the MRI machine hardware, signal-to-noise ratio, and image contrast of MRI, the imaging time commonly extends to several minutes. As a result, different parts of the collected kspace are acquired at different time instants. In the ideal scenario, the imaged object does not change during the period of the experiment, and the image calculated by inverse Fourier transformation is undistorted. However, in clinical MRI setups, this scenario is not usually guaranteed because of physiological and occasional voluntary 


\section{$25^{\text {th }}$ NATIONAL RADIO SCIENCE CONFERENCE (NRSC 2008)}

\section{C04 \\ March 18-20, 2008, Faculty of Engineering, Tanta Univ., Egypt}

patient motion and can be even impossible to realize for moving organs such as the heart and abdominal structures. Consequently, the constructed images suffer varying degrees of distortion depending on the characteristics of the imaging sequence and the severity of motion during the scan duration.

Motion artifacts can generally be classified into either intra-slice or inter-slice motion [1-2]. The first is the result of motion in between the acquisition of different portions of the k-space while the second is the result of motion in between acquisitions of the same slice. An illustration of these types of motion is shown in Fig. (1). The techniques in the literature often treated these types in completely different manners with several strategies to suppress each type independently. Given their underlying similarities, it might be advantageous to treat both problems simultaneously. In this work, we propose a new approach for suppressing motion artifacts from both types. The proposed method assumes rigid body motion and corrects for both its translational and rotational motion components without need for extra acquisitions.

\section{PREVIOUS WORK}

Several attempts to solve the problem of motion artifact in MRI have been reported in the literature. In general, the available techniques can be classified into four main categories. The first category attempts to suppress relative patient motion among different k-space lines within a given image through either through breath holding and chest strapping or by using cardiac and respiratory gating [3]. This minimizes the physiological component of motion between these lines at the expense of increased discomfort to the patient and/or significantly longer acquisition times. The second category uses averaging of different acquisitions to suppress the motion artifacts as well as to improve the signal-to-noise ratio (SNR) of the final image. This can be done by taking the average of the corresponding $\mathrm{k}$-space lines in a number of consecutive image acquisitions, or more generally by composing a weighted average of the two based on optimizing a certain objective function under given constraints $[3,14]$. The third category applies extra magnetic gradient lobes in the imaging sequence to eliminate the effects of motion through signal refocusing assuming a simple polynomial model for this motion $[16,19]$. This technique is used to minimize signal loss from moving blood and CSF within a given voxel [13]. Finally, the fourth category assumes simple forms of rigid body motion including translational and rotational components and corrects for them in a post-processing step. The motion in this category is estimated using external monitoring [20], navigator echo (only for translational motion) [1-2], symmetry constraints [9], motion periodicity constraint $[4,12]$, or through automated techniques[5,6,10-11,15,17-18]. The effect of translational motion can be suppressed by post-processing through modifying the phase of the k-space lines according to the $a$ priori knowledge about the motion [7-8,17].

In spite of the success these methods have met in some applications, they represent solutions to only a restricted class of artifacts and cannot generally be applied to more complex types of motion such as deformable body motions. Moreover, the convergence properties of automatic techniques are not generally guaranteed and therefore a general lack of robustness of these methods hindered their clinical use outside research facilities. As a result, if the patient moves significantly during the experiment, the motion artifact in the resultant images cannot be corrected. As a result, the scan has to be repeated at the expense of inefficient use of MRI machines and added discomfort to the patient. Moreover, this might not even be possible to tolerate in emergency cases. This also complicates the procedure of imaging moving organs such as the heart by adding the cardiac/respiratory gating, which again contributes to a significant prolongation of the examination time. Therefore, a technique for motion artifact suppression that does not impose any constraints on the current procedures while robustly constructing images that are free of motion artifact will have a rather profound impact on the current MRI technology and many of its clinical applications.

\section{METHODS}

Magnetic resonance imaging (MRI) is rapidly becoming a major modality because of its many promising and substantial capabilities for investigating various body organs, especially brain and extremities [1]. One of the problems encountered in MRI is patient movement during data acquisition, which typically takes several minutes. The motion causes artifacts in the reconstructed image, which for two-dimensional Fourier Transform (2DFT) imaging techniques appears as blurring and ghost repetitions of the moving structure. Patient motion can be classified according to its nature as rigid motion in which all the object points undergo the same motion and non-rigid motion such as physiological movements (respiratory, cardiac). In this paper, we consider the case of rigid motion, which is commonly encountered in MR images of head, brain and limbs. First, consider 


\section{March 18-20, 2008, Faculty of Engineering, Tanta Univ., Egypt}

the relation between the MR signal and the density distribution of the target in the imaging plane. This is given by,

$$
F\left(k_{x}, k_{y}\right)=\int_{-\infty}^{\infty} \int_{-\infty}^{\infty} f(x, y) \exp \left[-j 2 \pi\left(k_{x} x+k_{y} y\right)\right] d x d y,
$$

where $F\left(k_{x}, k_{y}\right)$ is the MR signal, $k_{x}$ and $k_{y}$ are the spatial frequency coordinates in the readout and phaseencoding directions, respectively, $f(x, y)$ is the density distribution of the nonmoving imaging target, and $x, y$ are horizontal and vertical coordinates in the imaging plane. In (1) it is seen that the MRI signal is the 2-D Fourier transform of $f(x, y)$. Considering the case when the k-space is acquired as consecutive bands, as in the case of segmented EPI and the proposed method, one can neglect the inter-band motion. This is true because the entire band is acquired during a single read-out period. Thus, planar rigid motion parameters during the acquisition can be regarded as a function of the band number. A planar rigid motion is the combination of translational and rotational motions. It is well known that the rotation of an object about the center of the image domain results in the same rotation of its k-space, while translational shift results in a linear phase term multiplied in the k-space [2]. Thus the effect of the motion can be written as,

$$
F_{d}\left(k_{x}, k_{y}\right)=\exp \left[-j 2 \pi\left(\delta_{x} k_{x}+\delta_{y} k_{y}\right)\right] . F_{\theta}\left(k_{x}, k_{y}\right) \text {. }
$$

Here $F_{d}\left(k_{x}, k_{y}\right)$ is the motion-distorted MRI signal and $\delta_{x}, \delta_{y}$, and $\theta$ are the translation in the x-direction, the translation in the y-direction and the rotation angle, respectively, and $F_{\theta}\left(k_{x}, k_{y}\right)$ is defined as,

$$
F_{\theta}\left(k_{x}, k_{y}\right)=F\left(k_{x} \cos \theta+k_{y} \sin \theta,-k_{x} \sin \theta+k_{y} \cos \theta\right)
$$

The motion correction problem is that of estimating the unknown motion parameters $\delta_{x}, \delta_{y}$, and $\theta$ and using these parameters to reconstruct an artifact-free image. An illustration of this motion when a k-space band is acquired is shown in Fig. (2).

Starting from the general assumption of rigid body motion, we consider the case when the acquisition of the k-space is in the form of overlapping bands of finite number of lines arranged in a rectilinear fashion to cover the k-space area of interest. We also assume that an averaging factor of at least two is desired (i.e., NEX $\geq 2$ ), to make the inter-slice motion problem nontrivial. Instead of acquiring a full $\mathrm{k}$-space of each image and then averaging the result, we propose a new acquisition strategy based on acquiring the k-space in consecutive bands having (100.(NEX-1)/NEX)\% overlap going from one end of the phase encoding direction to the other end. For example, when NEX is 2, the overlap will be $50 \%$. Fig. (3) illustrates the acquisition strategy proposed. Each band consists of a finite number of phase encoding lines acquired in a single shot so that the inter-band motion effect is limited. In case of no motion, this overlap provides the additional acquisitions required by the selected NEX value, while in the case when inter-band motion occurs; the proposed overlap provides the information that enables the determination of motion parameters as a generalization of the floating navigator echo [1].

The process of estimating the motion parameters is done in two steps: rotation estimation then 2-D translation estimation. From the geometry of the acquisition in the k-space in Fig. (2), the presence of rotation amounts to varying the area of overlap between the two consecutive bands or blades. Hence, given that this geometry is known a priori, if we compute a similarity measure between the areas of overlap at each possible rotation angle within a predetermined range of possible angles, it is possible to determine the rotation angle as the one having the highest similarity measure. The utilized method is based on computing the correlation coefficient of the magnitudes of the points in the overlap areas from the two different acquisitions such that,

$$
S M=\frac{\sum_{i, j \in \text { overlap }}\left(I_{1}(i, j)-m_{1}\right) \cdot\left(I_{2}(i, j)-m_{2}\right)}{\sqrt{\left(\sum_{i, j \in \text { overlap }}\left(I_{1}(i, j)-m_{1}\right)^{2}\right) \cdot\left(\sum_{i, j \in \text { overlap }}\left(I_{2}(i, j)-m_{2}\right)^{2}\right)}} .
$$

Here, $S M$ is the similarity measure correlation coefficient, $I_{1}$ is the magnitude of the first k-space acquisition, $I_{2}$ is the magnitude of the second $\mathrm{k}$-space acquisition, and $m_{1}$ is the mean of the k-space magnitudes within the overlap area of the first acquisition, while $m_{2}$ is the corresponding mean for the second acquisition. This allows for the similarity measure to be independent of the size of the overlapping area given that it is normalized to be within \pm 1 regardless of the number of points. It should be noted that a simple gridding procedure for one overlap 


\title{
$25^{\text {th }}$ NATIONAL RADIO SCIENCE CONFERENCE (NRSC 2008)
}

\author{
\begin{tabular}{l|l|}
4 & C04 \\
\hline
\end{tabular} \\ March 18-20, 2008, Faculty of Engineering, Tanta Univ., Egypt
}

area must be performed to align the data points with the second to have the same reference [23]. This is different from [21] where only an arc navigator is used in that using the whole overlap area makes the overall SNR of the estimation better. Hence, there is an expected trade-off between accuracy and computational complexity in these two techniques. Once the rotation is determined, it is straightforward to determine the 2-D translation using the floating navigator echo technique [1]. This process is done between each two consecutive bands with correction for translational motion for the most recently acquired band. On the other hand, the rotational motion can be corrected only during the reconstruction process given the sampling non-uniformity introduced into the k-space by this type of motion. The reconstruction includes an interpolation step to calculate the k-space data on a rectilinear grid using gridding algorithms [22-25]. A block diagram of the developed technique is shown in Fig. (4).

\section{RESULTS AND DISCUSSION}

The proposed algorithm was verified using simulated motion on numerical phantoms as well as real human head images acquired from a normal human volunteer inside a obtained on a Siemens Magnetom Trio 3T system. The volunteer was instructed to move during the data acquisition to induce motion artifacts. of an induced motion of a phantom. The acquisition parameters were as follows: imaging plane: sagittal, imaging sequence: fast spin echo, TR: 500ms, TE: 15ms, Matrix: 256x256, and ETL: 16, NEX: 2. Fig. (5) shows an example of rotation estimation using the proposed method. The correlation coefficient is computed and for different overlap angles and the angle at which maximum correlation is found is used as the rotation estimate. In Fig. (6), the results of applying the new method to numerically simulated axial brain slices are shown where a significant improvement is observed after the proposed method is applied. A wide range of motion parameters was simulated to test the accuracy of the proposed method and simulation of noisy data was performed to test the robustness of the solution under different SNR conditions. The results indicate that the new method is capable of detecting rotations with a mean error as low as \pm 0.1 degrees (which is more accurate than [21]) and translation with an error that is always less than \pm 0.2 of the pixel width. Given that the similarity measures used implicitly average the data, the technique was found to be robust against noise in most cases. Fig. (7) shows the results obtained from the real data. The arrows show several locations where the improvement is most visible. As can be noticed, the correction substantially improves the resolution of the image. However, ringing artifacts appear more prominent in the corrected image due to the problem of k-space voids shown in Fig. (8). Further investigation is needed to address this problem from both acquisition and reconstruction perspectives.

\section{CONCLUSIONS}

A new acquisition and processing strategy was proposed to suppress both intra-slice and inter-slice motion types more accurately based on the localization of overlapped k-space areas in between acquisitions. The new method was experimentally verified using simulated motion on numerical phantom as well as real images. The new technique has the advantage of not requiring additional acquisition time making its practical application possible.

\section{REFERENCES}

1. Y.M. Kadah, A. Abaza, A. Fahmy, A.M. Youssef, K. Heberlein, and X. Hu, "Floating navigator echo (FNAV) for in-plane 2D translational motion estimation," Magnetic Resonance in Medicine, Vol. 51, pp. 403-407, Feb. 2004.

2. R.L. Ehman and J.P. Felmlee, "Adaptive technique for high-definition MR imaging of moving structures," Radiology, Vol. 173, pp. 255-263, 1989.

3. M.L. Wood and R.M. Henkelman, "Suppression of respiratory motion artifacts in magnetic resonance imaging," Medical Physics, Vol. 13, No. 6, pp. 794-805, 1986.

4. T. Mitsa, K.J. Parker, W.S. Smith, A.M. Tekalp, and J. Szumowski, "Correction of periodic motion artifacts along the slice selection axis in MRI," IEEE Transactions on Medical Imaging, Vol. 9, No. 3, pp. 310-317, 1991.

5. M. Hedley, H. Yan, and D. Rosenfeld, "An improved algorithm for 2-D translational motion artifact correction," IEEE Transactions on Medical Imaging, Vol. 10, No. 4, pp. 548-553, 1991. 


\section{$25^{\text {th }}$ NATIONAL RADIO SCIENCE CONFERENCE (NRSC 2008)}

\section{March 18-20, 2008, Faculty of Engineering, Tanta Univ., Egypt}

6. R. Steagall, S. Amartur, and E.M. Haacke, "Correcting motion artifacts via a fast, iterative, POCS procedure," Proc. SMR Eighth Annual Meeting, 1990.

7. H.W. Korin, F. Farzaneh, R.C. Wright, and S.J. Riederer, "Compensation for effects of linear motion in MR imaging," Magnetic Resonance in Medicine, Vol. 12, pp. 99-113, 1989.

8. R.A. Zoroofi, Y. Sato, S. Tamura, H. Naito, and L. Tang, "An improved method for MRI artifact correction due to translational motion in the imaging plane," IEEE Transactions on Medical Imaging, Vol. 14, No. 3, pp. 471-479, 1995.

9. L. Tang, M. Ohya, Y. Sato, S. Tamura, H. Naito, K. Harada, and T. Kozuka, "Artifact cancellation in MRI due to phase encoding axis motion," Systems and Computers in Japan, Vol. 26, No. 1, pp. 88-97, 1995.

10. M. Hedley, H. Yan, and D. Rosenfeld, "A modified Gerchberg-Saxton algorithm for one-dimensional motion artifact cancellation in MRI," IEEE Transactions on Signal Processing, Vol. 39, No. 6, pp. 14281433, 1991.

11. M. Hedley, H. Yan, and D. Rosenfeld, "Motion artifact correction in MRI using generalized projections," IEEE Transactions on Medical Imaging, Vol. 10, No. 1, pp. 40-46, 1991.

12. M. Hedley and H. Yan, "Suppression of slice selection axis motion artifacts in MRI," IEEE Transactions on Medical Imaging, Vol. 11, no. 2, pp. 233-237, 1992.

13. J.L. Durek and P.M. Pattany, "Analysis of imaging axes significance in motion artifact suppression technique (MAST): MRI of turbulent flow and motion," Magnetic Resonance in Medicine, Vol. 7, pp. 251$263,1989$.

14. B. Madore and R.M. Henkelman, "A new way of averaging with applications to MRI," Medical Physics, Vol. 23, No. 1, pp. 109-113, 1996.

15. J.P. Felmlee, R.L. Ehman, S.J. Riederer, and H.W. Korin, "Adaptive motion compensation in MR imaging without use of navigator echoes," Radiology, Vol. 179, pp. 139-142, 1991.

16. P.M. Pattany, J.J. Phillips, L.C. Chiu, J.D. Lipcamon, J.L. Duerk, J.M. McNally, and Surya N. Mohapatra, "Motion artifact suppression technique (MAST) for MR imaging," Journal of Computer Assisted Tomography, Vol. 11, No. 3, pp. 369-377, 1987.

17. R.A. Zoroofi, Y. Sato, S. Tamura, and H. Naito, "MRI artifact cancellation due to rigid motion in the imaging plane," IEEE Transactions on Medical Imaging, Vol. 15, No. 6, pp. 768-784, 1996.

18. T.S. Sachs, C.H. Meyer, P. Irarrazabal, B.S. Hu, D.G. Nishimura, and A. Macovski, "The diminishing variance algorithm for real-time reduction of motion artifacts in MRI," Magnetic Resonance in Medicine, Vol. 34, pp. 412-422, 1995.

19. E.M. Haacke and G.W. Lenz, "Improving MR image quality in the presence of motion by using rephasing gradients," American Journal of Radiology, Vol. 148, pp. 1251-1258, 1987.

20. X. Hu, T.H. Le, T. Parrish, and P. Erhard, "Retrospective estimation and correction of physiological fluctuation in functional MRI," Magnetic Resonance in Medicine, Vol. 34, pp. 201-212, 1995.

21. H.M. Ahmed, R.E. Gabr, A.M. Youssef and Y.M. Kadah, "Combined intra- and inter-slice motion artifact suppression in magnetic resonance imaging," Proc. SPIE Medical Imaging 2003, San Diego, Feb. 2003

22. J.D. O'Sullivan, "A fast sinc function gridding algorithm for Fourier inversion in computer tomography," IEEE Transactions on Medical Imaging, Vol. MI-4, No. 4, pp. 200-207, 1985.

23. J.I. Jackson, C.H. Meyer, D.G. Nishimura, and A. Macovski, "Selection of a convolution function for Fourier inversion using gridding," IEEE transactions on Medical Imaging, vol. 10, no. 3, pp. 473-478, 1991.

24. C.H. Meyer, B.S. Hu, D.G. Nishimura, and A. Macovski, "Fast spiral coronary artery imaging," Magnetic Resonance in Medicine, vol. 28, pp. 202-213, 1992.

25. Y.M. Kadah, A. Fahmy, R. Gabr, K. Heberlein, and X. Hu, "Progressive magnetic resonance image reconstruction based on iterative solution of a sparse linear system," International Journal of Biomedical Imaging, Vol. 2006, pp. 1-9, 2006. 


\section{$25^{\text {th }}$ NATIONAL RADIO SCIENCE CONFERENCE (NRSC 2008)}

\begin{tabular}{l|l|l}
6 & C04 \\
\hline
\end{tabular}

March 18-20, 2008, Faculty of Engineering, Tanta Univ., Egypt
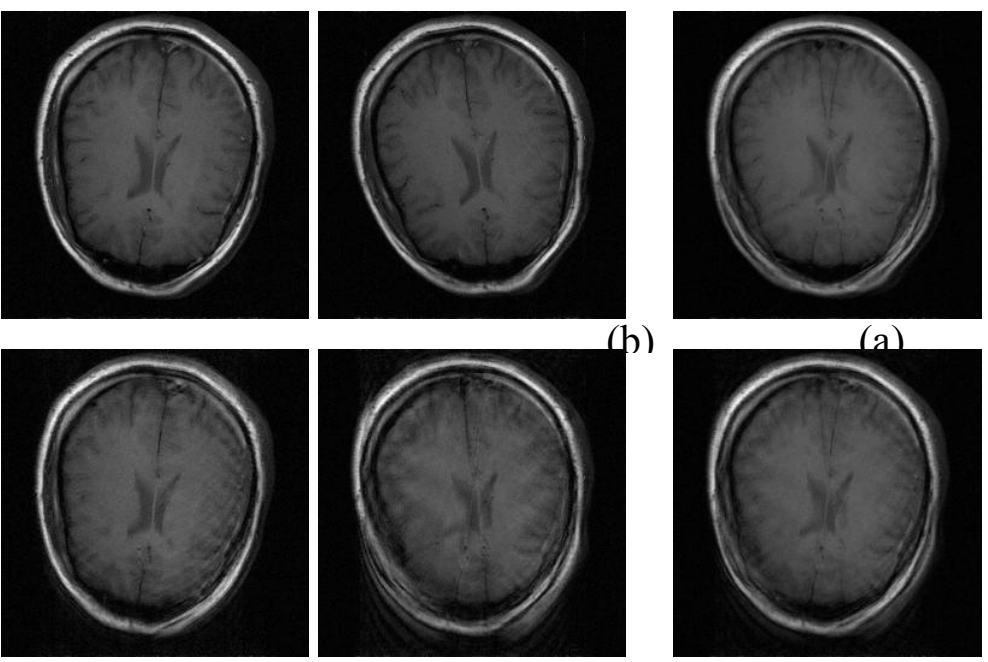

Fig. (1): Examples of motion artifacts: inter-slice motion artifact (above) results when images (a) and (b) are averaged to create the final image (c) on the system. On the other hand, if the same process is repeated with these individual images having an intra-slice motion (below), the result will be a more severe combined intra- and inter-slice motion artifact on image (c) below.
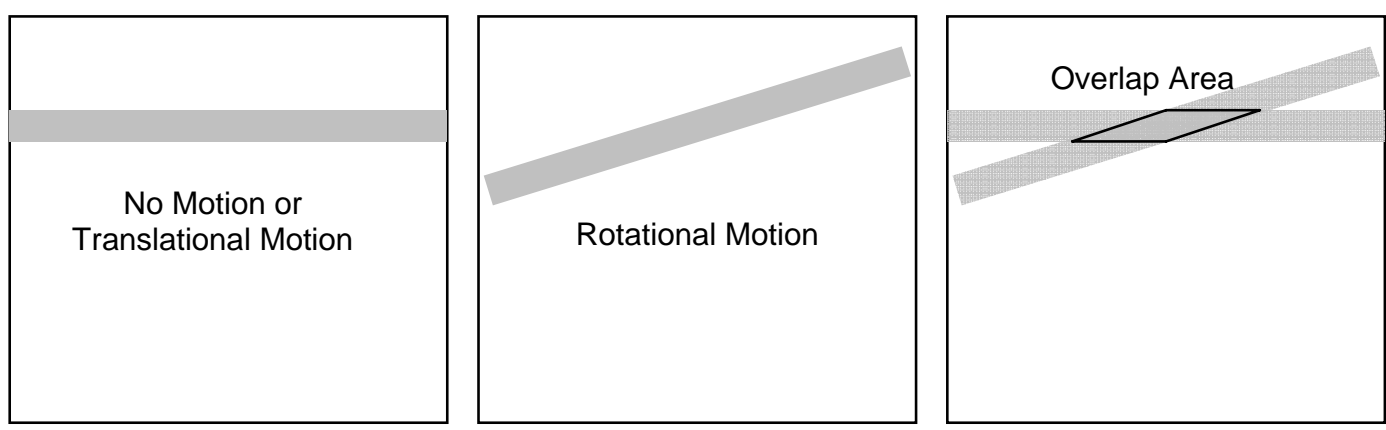

Fig. 2: Different cases in k-space band acquisition: no motion or translational motion (left), rotational motion while acquiring the same band (center). The overlap area between consecutive acquisitions in the worst case scenario is shown on the right. 


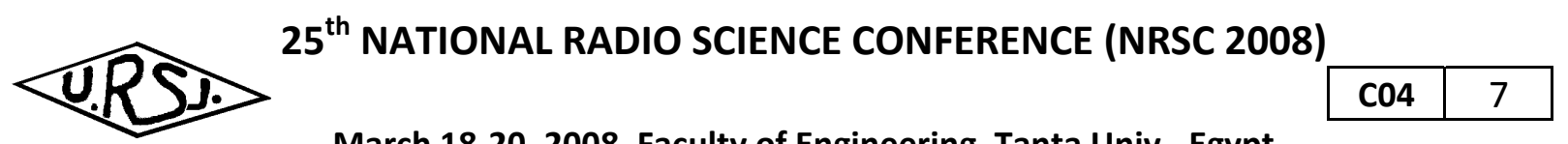

March 18-20, 2008, Faculty of Engineering, Tanta Univ., Egypt

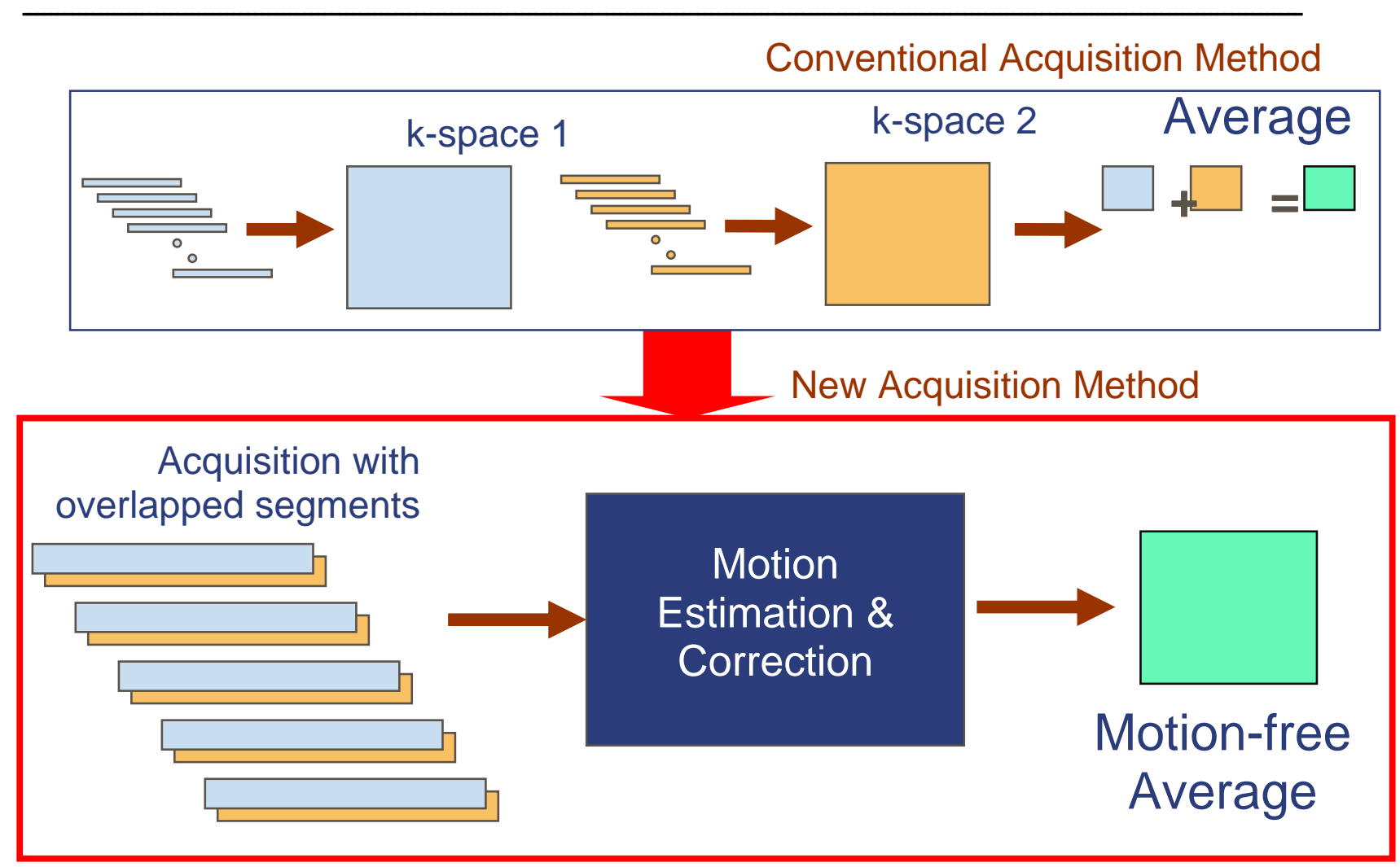

Fig. (3): Basic idea of the new method. Instead of doing the image acquisitions independently then performing the average, the acquisitions will be overlapped in such a way to allow the new overlap localization strategy to reconstruct an artifact-free image while maintaining the same acquisition time.

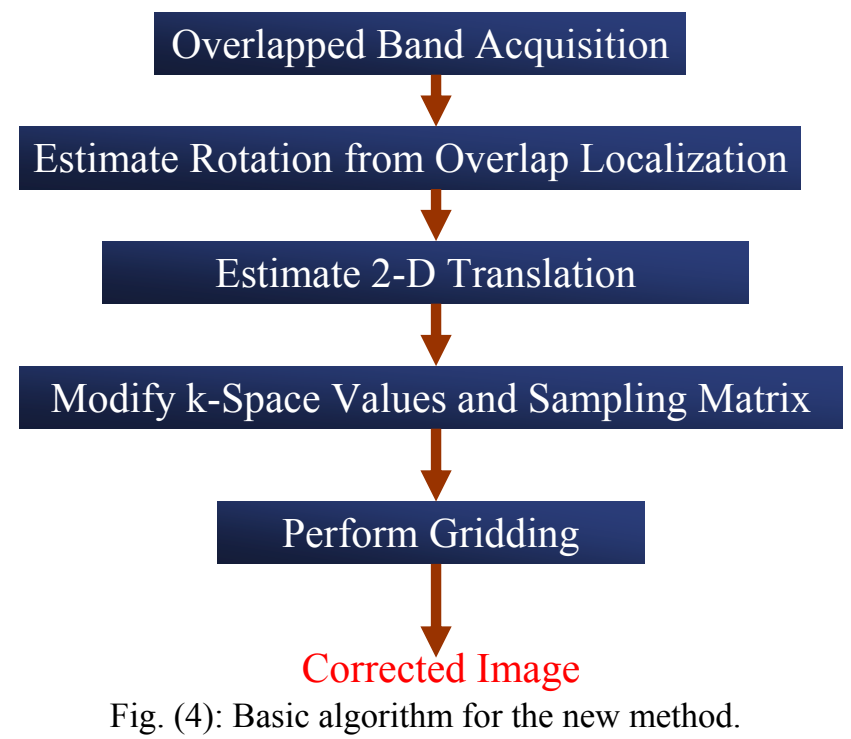




\section{$25^{\text {th }}$ NATIONAL RADIO SCIENCE CONFERENCE (NRSC 2008)}

\section{\begin{tabular}{l|l|}
8 & $\mathrm{CO4}$ \\
\hline
\end{tabular}}

March 18-20, 2008, Faculty of Engineering, Tanta Univ., Egypt
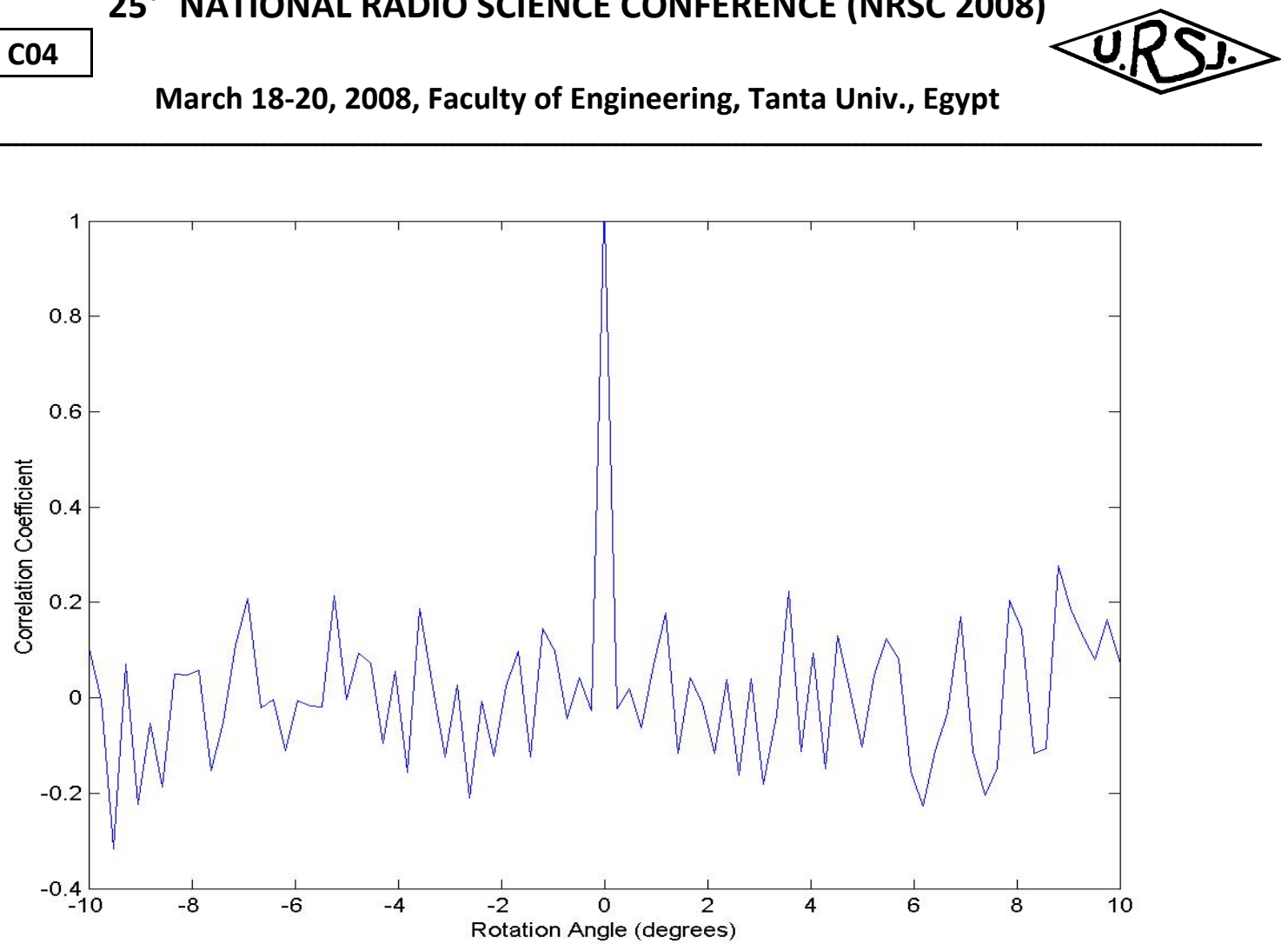

Fig. (5): Example of rotation estimation using the proposed method. The correlation coefficient is computed and for different overlap angles and the angle at which maximum correlation is found is used as the rotation estimate.

Motion-Free

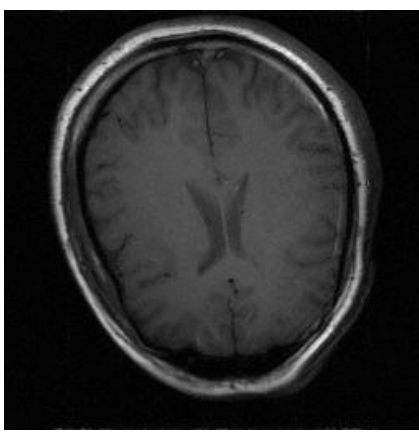

Motion-Corrupted

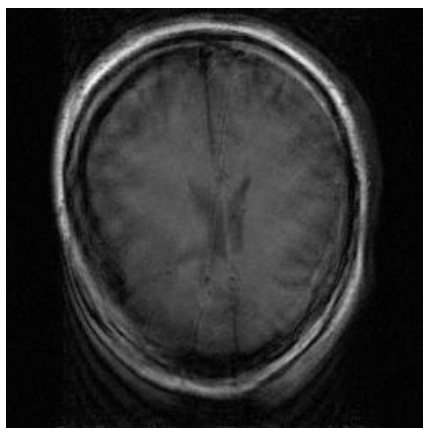

Corrected

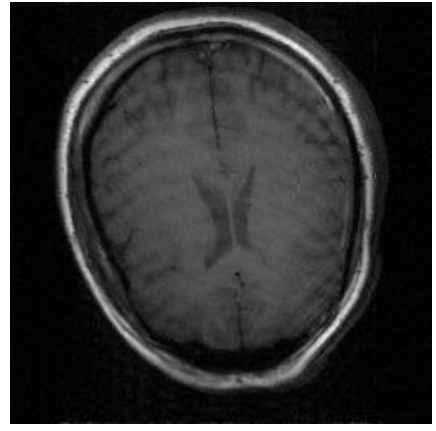

Fig. (6): Results of applying the new method to simulated data of axial brain slices of a human. As can be observed, substantial improvement is seen but ringing artifacts are visible in the corrected image due to possible k-space voids. 


\section{$25^{\text {th }}$ NATIONAL RADIO SCIENCE CONFERENCE (NRSC 2008)}

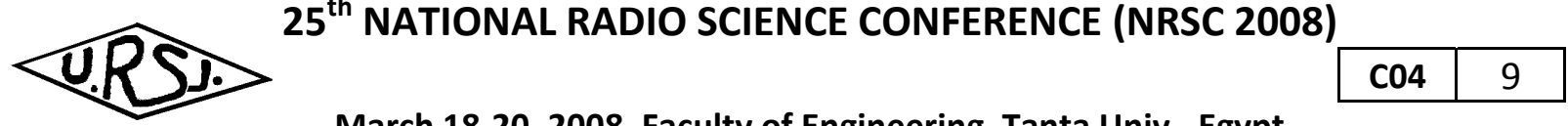

March 18-20, 2008, Faculty of Engineering, Tanta Univ., Egypt

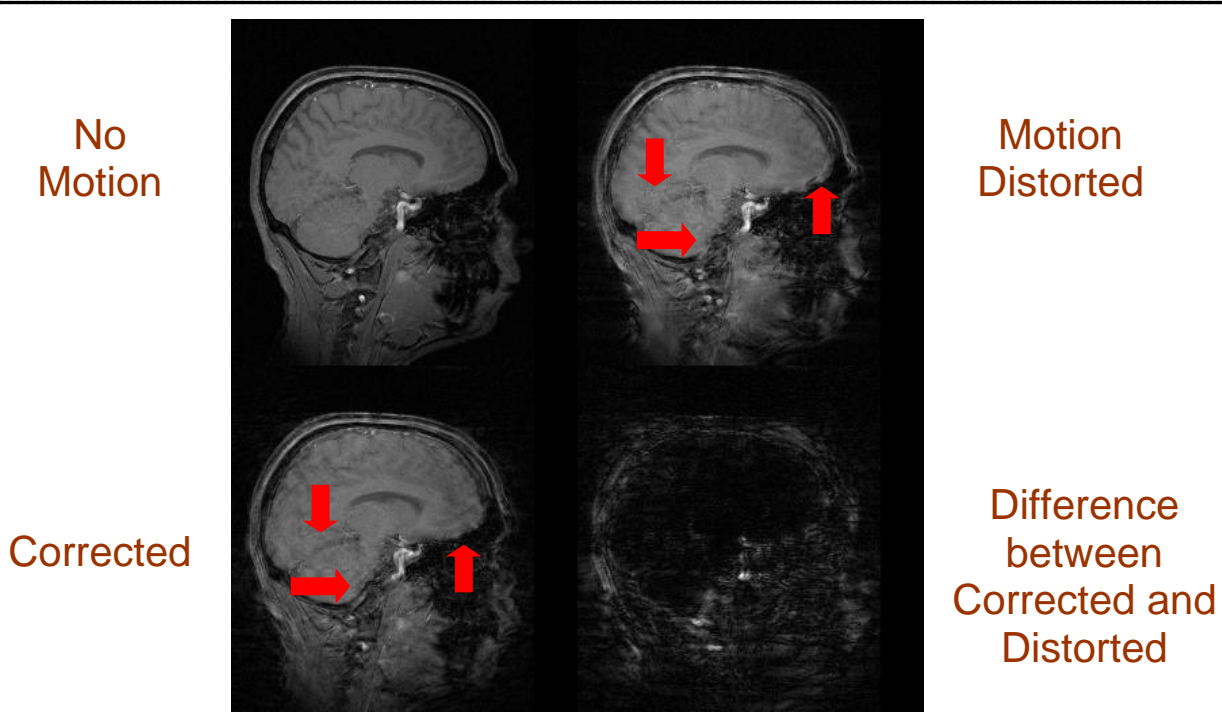

Fig. (7): Results of applying the new method to real data of sagittal brain slices of a human volunteer. Arrows point to areas in the image where correction is clearly visible. The difference image show the error removed by the new method.

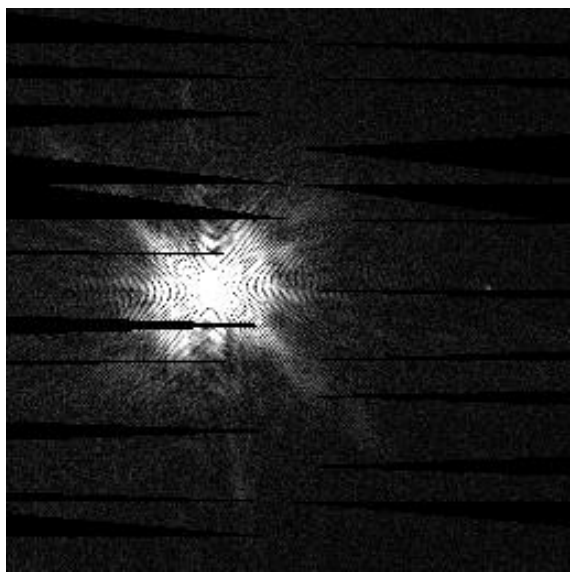

Fig. (8): Illustration of the problem of k-space voids where the rotated bands cause small scattered areas of the kspace to be missing thus leaving residual sampling artifacts in the reconstructed image after motion correction. 